\title{
Atomic ground state energies
}

\author{
R. K. Bhaduri AND M. Brack ${ }^{1}$ \\ Physics Department, McMaster Unicersity, Hamilton, Ont., Canada L8S 4MI \\ Received June 10, 1980
}

\begin{abstract}
It is demonstrated that atomic Hartree-Fock binding energies may be reproduced with great accuracy (within about four parts in a thousand) by a scaled model system in which the electrons are noninteracting, and are bound in a bare Coulomb potential.
\end{abstract}

\begin{abstract}
On démontre que les valeurs Hartree-Fock des énergies de liaison atomique peuvent être reproduites avec une grande précision (environ quatre partie sur mille) par un système de modèles à échelle dans lequel les électrons sont sans interaction et liés par un simple potentiel de Coulomb.
\end{abstract}

Can. J. Phys.. 58, 1684 (1980)

[Traduit par le journal]

In this article, we shall present a very simple analytical expression for the total binding energy of a neutral atom. We are aware that this quantity is only of peripheral interest in modern atomic physics, since the major contribution to the total energy comes from electrons in the innermost shells. Nevertheless, we report this work because our approach is novel, and the accuracy is better than a recently reported paper by Sucher (1) on the same subject. We demonstrate that the total ground state energy of any atom may be reproduced by a model system in which both the nuclear charge and the electron number are reduced by a scaling factor, and the electron-electron interactions switched off. The scaling factor is independent of $Z$, and its value can be guessed from theory. This model is rather different from the conventional effective charge approach $(2,3)$ in which only the nuclear charge, as seen by the electrons in each shell, is modified.

A neutral atom with $Z$ electrons is replaced by a model system of $Z_{0}$ noninteracting electrons bound in a bare Coulomb potential with nuclear charge $Z_{0}$. For such a system the closed shell interpolation formula for energy, in atomic units, is (4)

$$
\text { [1] }-E / Z_{0}^{2}=\left(\frac{3}{2} Z_{0}\right)^{1 / 3}\left(1+0.0636 Z_{0}{ }^{-2 / 3}\right)-\frac{1}{2}
$$

We take $Z_{0}=\beta Z$, where $\beta=0.831$ for all atoms with $Z>8$. Substituting this value of $Z_{0}$ in eq. [1], our expression for the ground state energy of an atom with atomic number $Z$ is

[2] $-E / Z^{2}=0.7432 Z^{1 / 3}\left[1+0.0719 Z^{-2 / 3}\right]$

In Table 1, the energies of some atoms as predicted by eq. [2] are compared with the corresponding (nonrelativistic) Hartree-Fock results. Our results

\footnotetext{
'Permanent address: Physics Department, University of Regensburg, 8400 Regensburg, W. Germany.
}

TABLE 1. Comparison of the HartreeFock energies $E_{\mathrm{HF}}$ with those predicted by eq. [2] for some neutral atoms. The energies are in atomic units, and divided by $Z^{2}$

\begin{tabular}{rcc}
\hline \hline$Z$ & $-E_{\mathrm{HF}} / Z^{2}$ & $-E / Z^{2}$, eq. [2] \\
\hline 8 & 1.169 & 1.168 \\
10 & 1.286 & 1.281 \\
13 & 1.431 & 1.425 \\
15 & 1.514 & 1.509 \\
18 & 1.626 & 1.623 \\
20 & 1.692 & 1.692 \\
25 & 1.840 & 1.846 \\
31 & 2.001 & 2.006 \\
36 & 2.124 & 2.125 \\
42 & 2.254 & 2.253 \\
49 & 2.391 & 2.389 \\
54 & 2.480 & 2.478 \\
65 & 2.657 & 2.656 \\
70 & 2.733 & 2.731 \\
75 & 2.806 & 2.802 \\
80 & 2.876 & 2.869 \\
87 & 2.969 & 2.960 \\
90 & 3.007 & 2.997 \\
95 & 3.069 & 3.058 \\
\hline
\end{tabular}

are generally more accurate than those of Sucher (1), considerably so in the region $8<Z<50$. Earlier, Scott (5) and Foldy (6) had given simple expressions for atomic binding energies which are also much less accurate than our eq. [2]. Scott derived his formula by adding to the leading Thomas-Fermi term corrections due to the exchange contribution, and a term to compensate for the diverging Thomas-Fermi density near the origin. Our approach in deducing eq. [2] will be entirely different.

The atomic Hamiltonian with $Z$ electrons may be written as

[3] $\mathscr{H}=\left(\sum_{i} t_{i}-\sum_{i} \frac{Z_{0}}{r_{i}}\right)+\left(\sum_{i<j} \frac{1}{r_{i j}}-\sum_{i} \frac{\left(Z-Z_{0}\right)}{r_{i}}\right)$ 
where the sum is over all electrons. We choose $Z_{0}=\beta Z$ so that the expectation value of the last term is zero, i.e.,

[4] $\frac{1}{2} Z(Z-1)\left\langle r_{i j}{ }^{-1}\right\rangle=Z^{2}(1-\beta)\left\langle r_{i}{ }^{-1}\right\rangle$ or

$$
(1-\beta)=\frac{1}{2}\left\langle r_{i j}{ }^{-1}\right\rangle /\left\langle r_{i}^{-1}\right\rangle
$$

for $Z \gg 1$. As before, we have assumed that $\beta$ is independent of $Z$. In the Thomas-Fermi approximation, which is exact for $Z \rightarrow \infty, \beta=6 / 7$, while Hartree-Fock calculations over a wide range of $Z$-values yield a value of $\beta$ close to $5 / 6$, thus substantiating our claim (1). Using the virial theorem, We may now write the Hartree-Fock energy (for $Z \gg 1)$ as

[5] $E_{\mathrm{HF}}=-\frac{1}{2} Z^{2} \beta\left\langle r_{i}^{-1}\right\rangle_{Z}^{\mathrm{HF}}$

In the above equation, the expectation value of $r_{i}{ }^{-1}$ is for a $Z$-electron system with HF wave functions. Our model equation [2], on the other hand, is for a system of $Z_{0}$ noninteracting electrons in a bare nuclear charge $Z_{0}$ :

[6] $E=-\frac{1}{2} Z_{0}{ }^{2}\left\langle r_{i}^{-1}\right\rangle Z_{0}^{\text {Bare }}$

Where the expectation value is now taken with hydrogenic wave functions. For [5] and [6] to be equal, we must have

[7] $\left\langle r_{i}{ }^{-1}\right\rangle_{Z}^{\mathrm{HF}}=\beta\left\langle r_{i}{ }^{-1}\right\rangle_{Z_{0}}^{\mathrm{Barc}}$

This relation should hold for any $Z \gg 1$, but we are unable to prove it for the general case. However, it is easy to verify it in the Thomas-Fermi limit $(Z \rightarrow \infty)$. In this case $(3)$

[8] $\left\langle r_{i}{ }^{-1}\right\rangle_{Z}^{\mathrm{HF}} \rightarrow\left\langle r_{i}{ }^{-1}\right\rangle_{Z}^{\mathrm{TF}}=4\left(\frac{2}{9 \pi^{2}}\right)^{1 / 3} \times 1.588 Z^{1 / 3}$ and
[9] $\left\langle r_{i}{ }^{-1}\right\rangle_{Z_{0}}^{\text {Bare }}=2(3 / 2)^{1 / 3}(\beta Z)^{1 / 3}$

Substituting these in eq. [7], we find $\beta=0.833 \approx 5 / 6$. This is the same value of $\beta$ obtained from eq. [4] in HF calculations, and explains why the HF binding energies are reproduced by the noninteracting scaled model. In fact, it is easy to verify eq. [7] numerically by calculating $\left\langle r_{i}^{-1}\right\rangle_{\mathrm{HF}}^{Z}$ using Table 2.3 in the book by Fischer (7). For the range of $Z$ values that we have considered, we find that the right-hand side of eq. [7] tends to overestimate $\left\langle r_{i}{ }^{-1}\right\rangle \mathrm{HF}$ by about 1 to $1.5 \%$ for $\beta=0.831$. This was the scaling factor used to fit the HF energies by eq. [2].

If we had used eqs. [5] and [8], with the TF value of $\beta=6 / 7$, we would have obtained the coefficient of $Z^{1 / 3}$ to be 0.7687 , which is correct in the limit of $Z \rightarrow \infty$. On the other hand, by using the empirical value of $\beta \approx 5 / 6$, we have shown that the energy may be written in the form of eq. [6], which is the content of our model. Our arguments, however, do not amuunt to a "derivation" of eq. [2], nor explain its extreme accuracy.

\section{Acknowledgements}

This work was supported by the Natural Sciences and Engineering Research Council of Canada. The authors thank Yuki Nogami and Peter Sutherland for useful discussions.

1. J. Sucher. J. Phys. B, 11, 1515 (1978).

2. J. C. Slater. Quantum theory of matter. McGraw Hill, New York, NY. 1951.p. 475.

3. R. O. Mueller, A. R. P. Rau, and L. Spruch. Phys. Rev. A, 8, 1186 (1973).

4. N. H. March. Self-consistent field in atoms. Pergamon Press, Oxford, England. 1975. pp. 49, 100.

5. J. M. C. Scott. Philos. Mag. 43, 859 (1952).

6. L. L. Foldy. Phys. Rev. 83, 397 (1951).

7. C. F. FISCHER. The Hartree-Fock method for atoms. John Wiley, New York, NY. 1977. p. 27. 